\title{
On Scattering of a Vector Cylindrical Wave by an Axisymmetric Semitransparent Reflector
}

\author{
Kirill Klionovski, Atif Shamim \\ Integrated Microwave Packaging Antennas and Circuits Technology Laboratory \\ King Abdullah University of Science and Technology (KAUST) \\ Thuwal, Kingdom of Saudi Arabia \\ kirill.klionovski@kaust.edu.sa, atif.shamim@kaust.edu.sa
}

\begin{abstract}
Semitransparent surfaces are used in antenna reflectors to obtain the required shape of the radiation pattern. The task of transparency optimization can be simplified with analytical formulae. In this paper, we investigate the scattering of a vector cylindrical wave by a semitransparent half-plane and axisymmetric reflector. The profile of the reflector can be concave, convex, or concavo-convex. We derive some asymptotic formulae for the radiation pattern of the scattering task. In this paper, we present the numerical results calculated through the asymptotic formulae. These results have also been verified through the method of moments.
\end{abstract}

Keywords—cylindrical wave; semitransparent surface; scattering.

\section{INTRODUCTION}

Semitransparent ground planes or reflectors with resistive edges can help with the suppression of back radiation or formation of a sharp main beam with low sidelobe levels. To analyze such scatterers, two-dimensional (2D) models of scattering of cylindrical waves by semitransparent strips and parabolic, hyperbolic, and elliptic reflectors have been used in [1-5]. In those papers, the scattering tasks have been solved by numerical solutions of integral equations. However, for the purpose of transparency optimization to obtain the required shape of the radiation pattern, analytical formulae can be advantageous as they are more representative of the physical phenomenon, thus provide more insight.

In this paper, we derive analytical asymptotic formulae for a 2D task of scattering of a vector cylindrical wave by a semitransparent (in particular, perfectly conducting) half-plane and reflector, which are pertinent to the transparency optimization issue. To estimate the accuracy of the formulae, we compare the asymptotic solution with a numerical solution of the scattering task obtained through the method of moments (MoM).

\section{ASYMPTOTIC SOLUTION For A HALF-PLANE}

Let us consider the cylindrical wave scattering by a semitransparent half-plane, as shown in Fig. 1(a). The source of the cylindrical wave is located at the point $(x=h, y=0)$ and is at a distance $L_{h p}$ from the edge of the half-plane along the $y$-axis. The radiation pattern of the cylindrical wave has the azimuthal $H_{\varphi}(\varphi)$ and axial $H_{z}(\varphi)$ components of the magnetic field intensity vector in free space in cylindrical coordinates $(\rho, \varphi, z)$. The semitransparent surface is characterized by two reflection

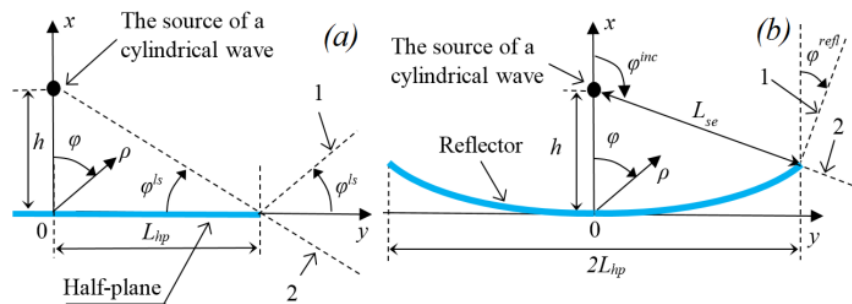

Fig. 1. The source of a cylindrical wave above $(a)$ a half-plane and $(b)$ a concave reflector: 1 - light-shadow boundary of the reflected wave, 2 - lightshadow boundary of the incident wave.

coefficients $\mathrm{R}_{\perp, \|}(y)$, and two transmission coefficients $\mathrm{T}_{\perp, \|}(y)$ (for waves with perpendicular $(\perp)$ or parallel $(\|)$ orientation of the magnetic field intensity vector relative to the edge of the half-plane). In general case, these coefficients are complex numbers with arguments to be within the range of $[-\pi / 2 ; \pi / 2]$.

The simplest asymptotic solution of the scattering task can be obtained in the geometric optics (GO) approximation:

$$
\begin{aligned}
& H_{\varphi, z}^{G O}(\varphi)=H_{\varphi, z}(\varphi) e^{i k h \cos \varphi} \chi\left(\varphi^{i n c}-\varphi\right)+ \\
& +\xi \mathrm{R}_{\perp, \|}(h \tan \varphi) H_{\varphi, z}(\pi-\varphi) e^{-i k h \cos \varphi} \chi\left(\varphi^{r e f l}-\varphi\right)+ \\
& +\mathrm{T}_{\perp, \|}(h \tan (\pi-\varphi)) H_{\varphi, z}(\varphi) e^{i k h \cos \varphi} \chi\left(\varphi-\varphi^{i n c}\right),
\end{aligned}
$$

where, $k=2 \pi / \lambda ; \lambda$ is the radiation wavelength; $i=\sqrt{-1} ; \xi$ is equal to -1 and 1 for the azimuthal and axial component of the magnetic field intensity vector, respectively; $\varphi^{i n c}=\pi / 2+\varphi^{l s}$; $\varphi^{r e f l}=\pi / 2-\varphi^{l s} ; \chi(\varphi)$ is the Heaviside function; $\varphi^{l s=} \arctan \left(h / L_{h p}\right)$.

To get the asymptotic solution in the shady region, let us follow the geometrical theory of diffraction (GTD) method. The GTD pattern is calculated as a sum of GO pattern and the pattern of an edge wave. For the case of a plane wave incident at the angle $\varphi^{l s}$ onto a semitransparent edge, the radiation pattern of an edge wave has the following empirical representation [6]:

$$
H_{\perp, \|}^{S}(\varphi)=\frac{-0.5\left(1-\mathrm{T}_{\perp, \|}\left(L_{h p}\right)\right)}{\cos \left(\frac{\pi / 2-\varphi^{l s}+\varphi}{2}\right)}-\frac{0.5 \xi \mathrm{R}_{\perp, \|}\left(L_{h p}\right)}{\cos \left(\frac{\pi / 2+\varphi^{l s}+\varphi}{2}\right)} .
$$

The GTD pattern $\left(H^{G T D}\right)$ of the scattering of the cylindrical wave by a semitransparent half-plane is expressed through the function $\tilde{F}(x)=-e^{-i x^{2}} /(2 x \sqrt{i \pi})$ that is the first term of the asymptotic expansion of the Fresnel integral 


$$
\begin{aligned}
& F(x)=-\operatorname{sign}(x) \sqrt{\frac{i}{\pi}} \int_{-\infty}^{-s i g n(x) x} e^{-i t^{2}} d t: \\
& H_{\varphi, z}^{G T D}(\varphi)=H_{\varphi, z}^{G O}(\varphi)+H_{\varphi, z}\left(\varphi^{i n c}\right)\left[\left(1-\mathrm{T}_{\perp, \|}\left(L_{h p}\right)\right) \times\right. \\
& \left.\times e^{i k h \cos \varphi} \tilde{F}\left(s_{1}^{r}(\varphi)\right)+\xi \mathrm{R}_{\perp, \|}\left(L_{h p}\right) e^{-i k h \cos \varphi} \tilde{F}\left(s_{1}^{m}(\varphi)\right)\right],
\end{aligned}
$$

where arguments of $\tilde{F}$ are calculated as follow:

$$
\begin{aligned}
& s_{1}^{r}(\varphi)=\sqrt{2 k L_{s e}} \sin \left(\left(\varphi^{i n c}-\varphi\right) / 2\right), \\
& s_{1}^{m}(\varphi)=\sqrt{2 k L_{s e}} \sin \left(\left(\varphi^{r e f l}-\varphi\right) / 2\right) .
\end{aligned}
$$

For the half-plane: $L_{s e}=\sqrt{L_{h p}^{2}+h^{2}}$. Asymptotic solution (3) is inapplicable near the light-shadow boundaries where it has singularities in the expression of the pattern of the edge wave (2). To compensate the singularities, let us follow the uniform geometrical theory of diffraction (UGTD) method by multiplying each function $\tilde{F}(x)$ in (3) by the transition function [7]:

$$
F^{\text {trans }}(x)=2 i e^{i x^{2}} \operatorname{sign}(x) x \int_{-\infty}^{-\operatorname{sign}(x) x} e^{-i t^{2}} d t \text {. }
$$

As a result, the UGTD pattern $\left(H^{U G T D}\right)$ is expressed in terms of the GO pattern, the discontinuities of which are smoothened by the Fresnel integrals:

$$
\begin{aligned}
& H_{\varphi, z}^{U G T D}(\varphi)=H_{\varphi, z}^{G O}(\varphi)+H_{\varphi, z}\left(\varphi^{i n c}\right)\left[\left(1-\mathrm{T}_{\perp, \|}\left(L_{h p}\right)\right) \times\right. \\
& \left.\times e^{i k h \cos \varphi} F\left(s_{1}^{r}(\varphi)\right)+\xi \mathrm{R}_{\perp, \|}\left(L_{h p}\right) e^{-i k h \cos \varphi} F\left(s_{1}^{m}(\varphi)\right)\right] .
\end{aligned}
$$

\section{AsYMPtotic SOLUTION For A REFLECTOR}

Let us consider the scattering of the field of the same source of a cylindrical wave located at distance $h$ from the centre of an axisymmetric semitransparent reflector. A smooth and slowly varying function $x=f_{R}(y), y \in\left[0 . . L_{h p}\right]$, determines the profile of the reflector. We assume that both the profile and the distribution of reflection and transmission coefficients are symmetric relative to the $x$-axis. The reflector can be concave (Fig. 1(b)), convex, or concavo-convex. The source illuminates fully the surface of the reflector. The UGTD pattern of the source above the reflector is determined through a GO pattern of the scattering task $H^{G O}{ }_{-}$, an eikonal of the wave reflected from the reflector $S_{\text {refl, }}$, and the reflection $\mathrm{R}_{\perp, \|}^{\text {edge }}$ and transmission $\mathrm{T}_{\perp, \|}^{\text {edge }}$ coefficients at the edge of the reflector:

$$
\begin{aligned}
& H_{\varphi, z}^{U G T D}(\varphi)=H_{\varphi, z}^{G O_{-} R}(\varphi)+H_{\varphi, z}\left(\varphi^{i n c}\right)\left[\left(1-\mathrm{T}_{\perp, \|}^{e d g e}\right) e^{i k h \cos \varphi} \times\right. \\
& \left.\times F\left(s_{1}^{r}(\varphi)\right)+\xi \mathrm{R}_{\perp, \|}^{e d g e} e^{-i k S_{r e f}(\varphi)} F\left(s_{1}^{m}(\varphi)\right)\right]+H_{\varphi, z}\left(-\varphi^{i n c}\right) \times \\
& \times\left[\left(1-\mathrm{T}_{\perp, \|}^{e d g e}\right) e^{i k h \cos \varphi} F\left(s_{2}^{r}(\varphi)\right)+\xi \mathrm{R}_{\perp, \|}^{e d g e} e^{-i k S_{r e f l}(\varphi)} F\left(s_{2}^{m}(\varphi)\right)\right], \\
& s_{2}^{r, m}(\varphi)=\left\{\begin{array}{ll}
s_{1}^{r, m}(-\varphi), & 0 \leq \varphi<\pi / 2 \\
s_{1}^{r, m}(2 \pi-\varphi), & \pi / 2 \leq \varphi \leq \pi
\end{array} .\right.
\end{aligned}
$$

\section{NUMERICAL RESULTS}

Let us consider a line current flown along the $z$-axis, which is located at the point $x=0.25 \lambda$ above a corner reflector with the profile of $f_{R}(y)=0.1 y, L_{h p}=2 \lambda$. Such $z$-oriented electric or magnetic current has unidirectional $\left(H_{\varphi, z}=1\right)$ azimuthal or axial component of the radiation pattern, respectively. We consider a perfectly conducting reflector $\left(\mathrm{R}_{\perp, \|}=1, \mathrm{~T}_{\perp, \|}=0\right)$, and a semitransparent one with the following transparency: $\mathrm{R}_{\perp, \|}(y)=1 /\left(1+0.58 y / L_{h p}\right), \quad \mathrm{T}_{\perp, \|}(y)=1-\mathrm{R}_{\perp, \|}(y)$. Fig. 2 shows radiation patterns calculated through formula (7). For the perfectly conducting case, we calculate radiation patterns numerically through EDEM software [8], which calculates scattering of electromagnetic waves by the MoM.

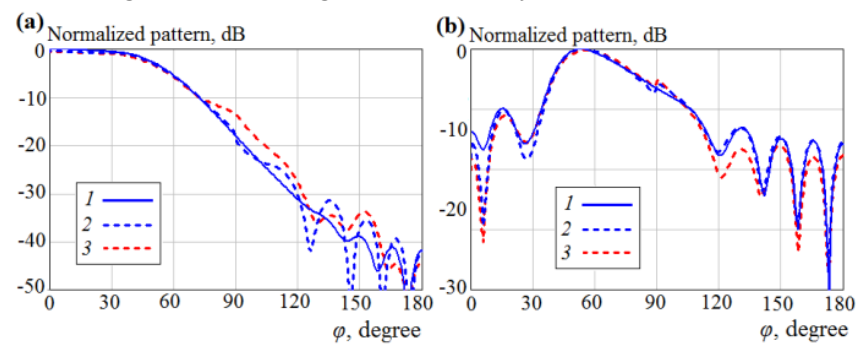

Fig. 2. Radiation pattern of (a) the electric line source and (b) the magnetic line source above the corner reflector: 1 - numerical solution for perfectly conducting reflector; 2 and 3 - asymptotic solution for perfectly conducting and semitransparent reflector, respectively.

\section{CONCLusions}

In this paper, we present an asymptotic solution of the scattering of a vector cylindrical wave by a semitransparent reflector. The asymptotic solution is based on the use of an edge wave for a semitransparent edge and the UGTD method. It is expressed as a GO approximation smoothened by the Fresnel integrals.

\section{REFERENCES}

[1] M. F. Otero and R. G. Rojas, "Resistive treatment to reduce edge diffraction from large wedge-shaped objects and planar antennas," Radio Science, vol. 32, no. 5, pp. 1745-1759, Sept.-Oct. 1997.

[2] A. I. Nosich, V. B. Yurchenko, and A. Altintas, "Numerically Exact Analysis of a Two-Dimensional Variable-Resistivity Reflector Fed by a Complex-Point Source," IEEE Trans. Antennas Propag., vol. 45, no. 11, pp. 1592-1601, Nov. 1997.

[3] T. Oguzer, A. I. Nosich, and A. Altintas, "Analysis of an Arbitrary Conic Section Profile Cylindrical Reflector Antenna, H-Polarization Case," IEEE Trans. Antennas Propag., vol. 52, no. 11, pp. 3156-3162, Nov. 2004.

[4] T. Oguzer, A. Altintas, A. I. Nosich, "Integral equation analysis of an arbitrary-profile and varying-resistivity cylindrical reflector illuminated by an E-polarized complex-sourcepoint beam," J. Opt. Soc. Amer., vol. 26, no. 7, pp. 1525-1532, July 2009.

[5] T. Oguzer, A. Altintas, A. I. Nosich, "Analysis of the elliptic-profile cylindrical reflector with a non-uniform resistivity using the complex source and dual-series approach: H-polarization case," Optical and Quantum Electronics, vol. 45, no. 8, pp. 797-812, Aug. 2013.

[6] J. Shmoys, "Diffraction by a Half-Plane with a Special Impedance Variation," IRE Trans. Antennas Propag., vol. 7, no. 5, pp. 88-90, Dec. 1959.

[7] D. A. McNamara, C. W. I. Pistorius, J. A. G. Malherbe, Introduction to the uniform geometrical theory of diffraction. Norwood, MA, USA: Artech house. 1990.

[8] http://edem3d.ru 